\title{
Acoustic and visual display of photons: a handheld demonstration device
}

\author{
T L Dimitrova ${ }^{1,2}$, A Lechkov ${ }^{3}$, Ts Grigorova ${ }^{3}$ and A Weis ${ }^{2}$ \\ ${ }^{1}$ Faculty of Physics, University of Plovdiv, Tzar Assen Street 24, 4000 Plovdiv, Bulgaria \\ ${ }^{2}$ Department of Physics, University of Fribourg, CH-1700 Fribourg, Switzerland \\ ${ }^{3}$ Departments of Electronics, Technical University Sofia-Branch Plovdiv, 61 Street, \\ Petersburg Building, 4000 Plovdiv, Bulgaria \\ E-mail: tldimitrova@abv.bg
}

\begin{abstract}
A handheld device for rendering the signals produced by individual photons is presented in this paper. Electric pulses produced by individual photons in a photomultiplier are made audible by a small loudspeaker built into the housing. Output connectors allow the pulse signals to be displayed by an external amplifier/loudspeaker system, an oscilloscope, or to be further processed by pulse counting electronics. The device is intended to be used for lecture (or public) demonstrations of the discrete nature of light.
\end{abstract}

\section{Introduction}

Among all ideas in physics, the concept of the physical nature of light has had one of the most intriguing and dramatic developments. Following many centuries of rather philosophical discussions, scientific debate about the nature of light took off in the 17th century. The well-known debate between the corpuscular theory of Isaac Newton, on the one hand, and the contradictory wave front theory of Christian Huygens, on the other hand, has been the focus of scientific discussion for several centuries. Huygens presented his Treatise on Light in 1690, but the book was published only 12 years later $[1]^{4}$. Newton started his experiments on light in the mid-1660s [2] and it was only in 1704, after the death of his main opponent Huygens, that he published his treatise Opticks [2]. Despite the criticism of Huygens, Hook and others, Newton's theory was the leading point of view for more than a century. Later, the works of Fresnel, Arago and Young gave more satisfying proofs of wave theory, which were supported by the theory of electromagnetic waves by Maxwell and their experimental discovery by Hertz.

A crucial revision of classical physics was needed soon after Young's experimental demonstration of the wave nature

\footnotetext{
4 The author states in the preface that he communicated his treatise to the 1
} French Royal Academy of Science in France in 1678. of light by the observation of interference in his famous double-slit experiments. The classical wave theory was able to explain neither blackbody radiation nor the photoelectrical effect. The revolutionary hypothesis of Max Planck (1899) that light is composed of discrete energy packages (quanta) was further promoted by Einstein (1905). This finally led to a unification of the two contradicting theories of light by Bohr in terms of the concept of wave-particle duality which laid the foundation of modern quantum physics (for a general discussion of the Einstein-Bohr debate, see [3]). In the quantum concept, light is a stream of elementary massless particles (photons) with energy and momentum that are described by an associated quantum mechanical wave function that explains the interference phenomena.

Although controversially debated in recent times [4], the photoelectric effect is usually introduced in elementary quantum mechanics courses to illustrate the particle nature of light. Two of us (TLD and AW) have developed several demonstration experiments on wave-particle duality and quantum erasing [5-7]. In those experiments a photomultiplier (PM) is used to detect light quanta from a strongly attenuated green $(\lambda=532 \mathrm{~nm})$ laser pointer after their passage through a (double-slit, Mach-Zehnder or Michelson) interferometer. The PM pulses, produced by individual quantum events, are rendered visually on an oscilloscope and acoustically as clicks of a loudspeaker. 


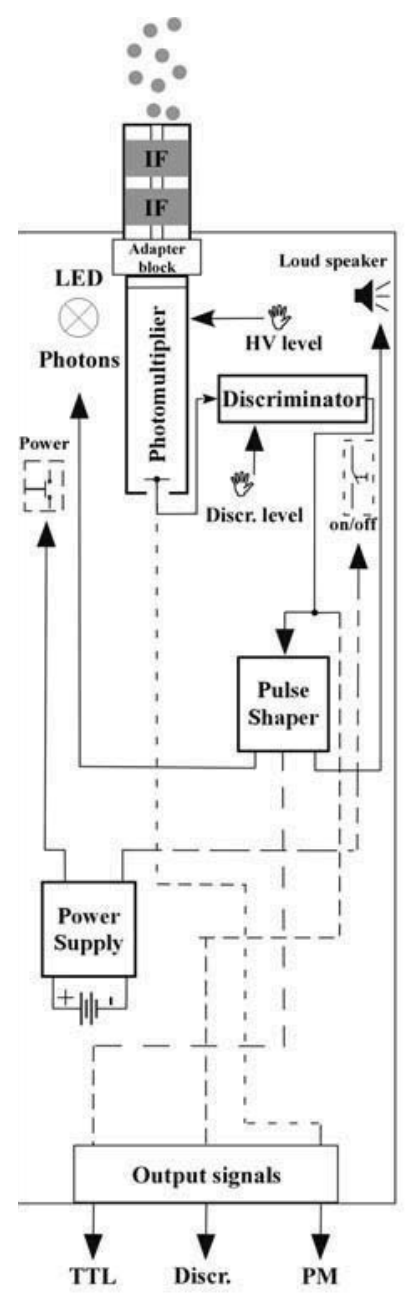

a)

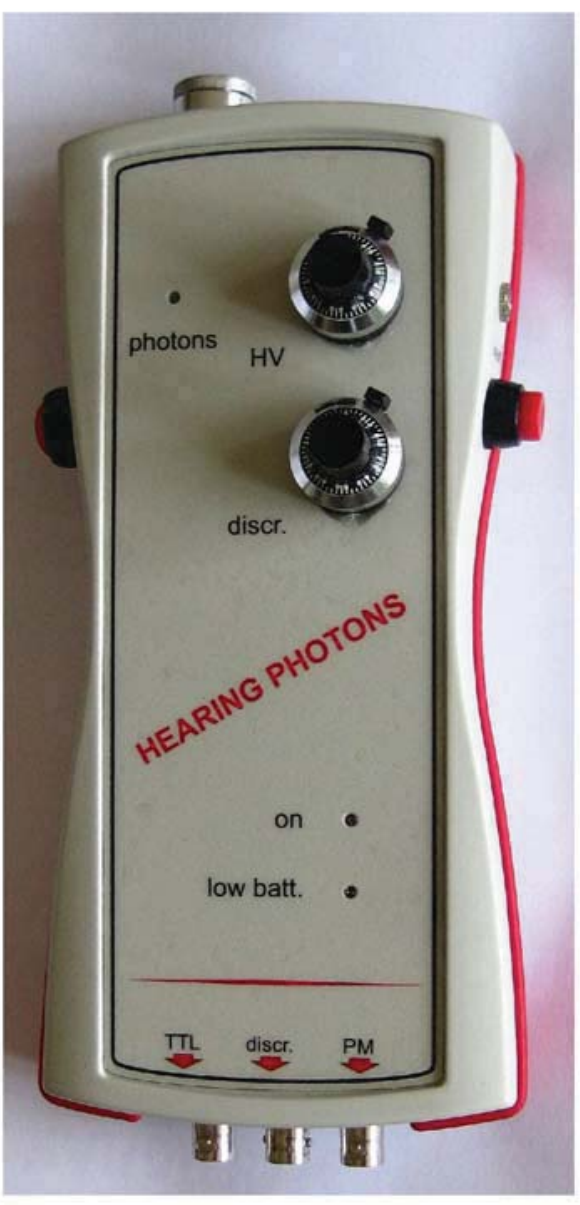

b)

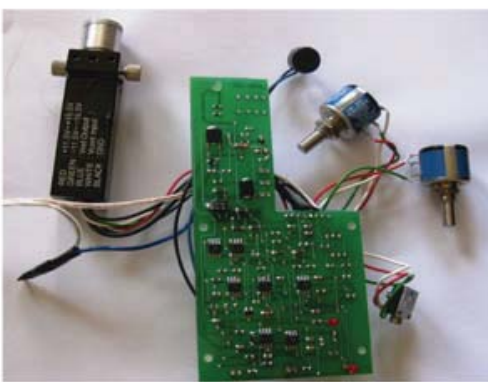

c)

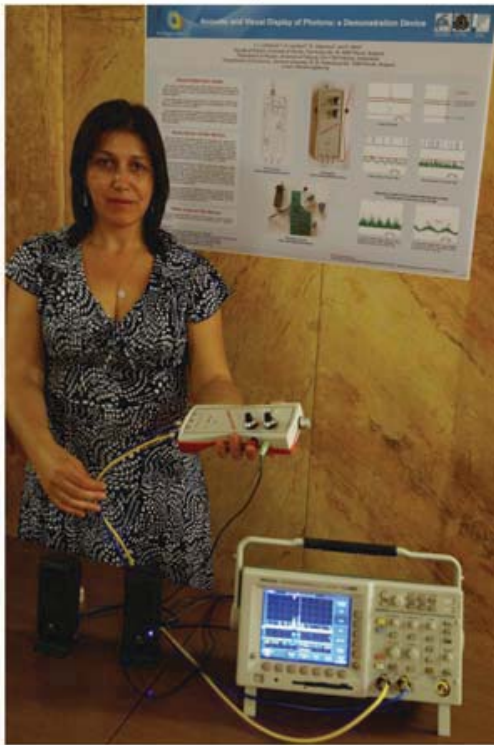

d)

Figure 1. Hearing photons device: (a) block scheme; (b) photograph; (c) electronic circuit; (d) presentation of the device by TLD at Photonica 2011.

Here we have explored the same principle for designing a handheld device that can be used in lecture demonstrations or in other public events for illustrating the discrete nature of light. We will refer to our apparatus as the 'hearing photons' device.

\section{Technical description of the 'hearing photons' device}

The 'hearing photons' device is based on a compact $(22 \times$ $22 \times 51 \mathrm{~mm}^{3}$ ) PM (Hamamatsu-type H10722-01), which contains a controllable high-voltage supply and a pulse preamplifier $^{5}$. The PM operates on supply voltages of $\pm 5 \mathrm{~V}$, which are delivered by rechargeable batteries. Figure 1(a) shows a block diagram of the device. A simple electronic discriminator circuit allows the analogue pulses of varying amplitudes produced by the PM to be converted into digital transistor-transistor logic (TTL) pulses.

Because of inherent statistical fluctuations of the (multistage) electronic amplification process in the PM, the analogue output pulses of the PM have a Poissonian

\footnotetext{
5 http://sales.hamamatsu.com/index.php?id=13226587
}

amplitude distribution. This is an unfortunate feature from a didactic point of view, which-to our experience-leads to confusion among young students who wonder why the detection of individual, nominally identical photons leads to pulses with amplitudes that vary so strongly. In order to circumvent an explanation of this effect during presentations (although interesting per se, it may distract from the main message that the demonstration wants to convey), one gives all pulses corresponding to the detection of an individual photon a uniform identical shape. This is achieved by an electronic comparator, which compares the amplitude of the analogue pulse with a (adjustable) reference voltage level (the discriminator level), and which produces a (uniform) pulse only in those cases, in which the analogue pulse exceeds the discriminator level. The discriminator level is adjusted experimentally: it should be sufficiently low so that a maximum number of photons can be 'caught' and at the same time sufficiently high so that electronic noise of the preamplifier does not produce accidental pulses. The uniform pulse level is usually chosen to be compliant with the TTL norm (a logical ' 0 ' corresponding to positive voltages between 0 and $0.8 \mathrm{~V}$, and a logical ' 1 ' corresponding to voltages between 2 and $3-5 \mathrm{~V}$ ). 

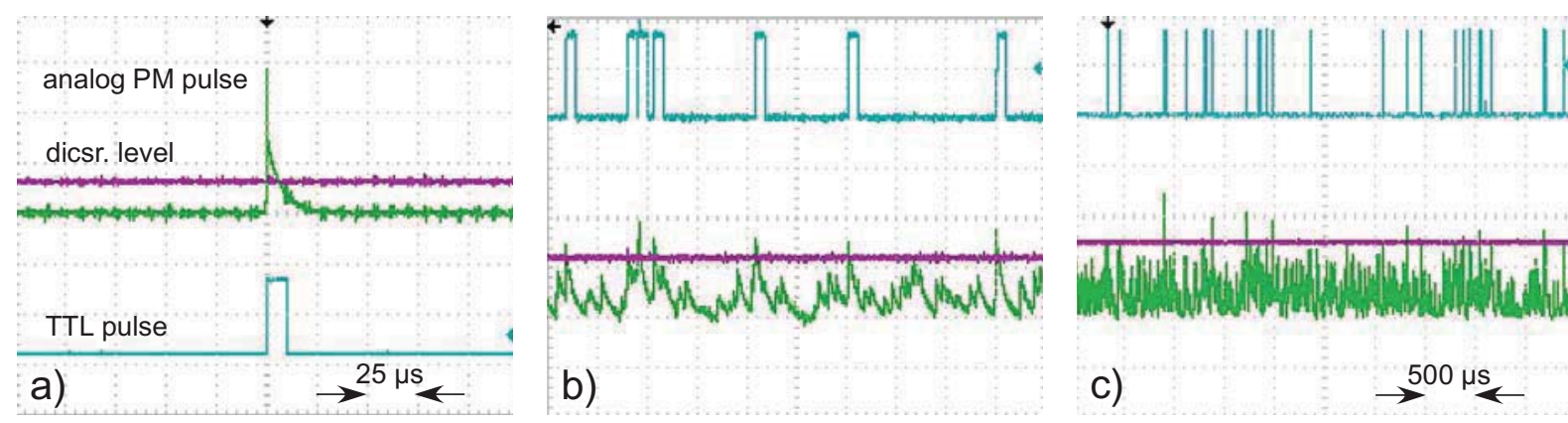

Figure 2. Oscilloscope traces of the PM, TTL and discriminator level signals: (a) single PM pulse; (b), (c) typical pulse trains recorded during 0.5 and $5 \mathrm{~ms}$, respectively.

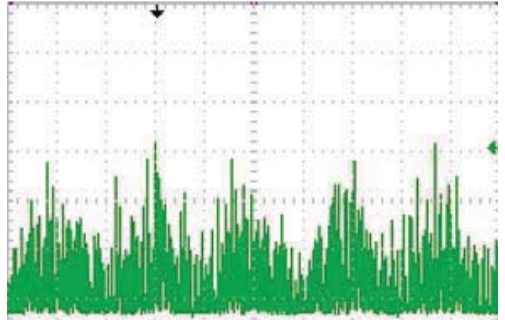

a)

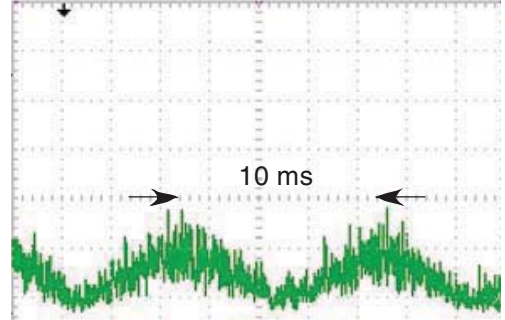

b)

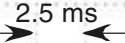

Figure 3. Detection of light from a distant fluorescence lamp (via the green resonance line of Hg): (a) single oscilloscope trace showing individual photons with indication of a periodic bunching of the photon rate; (b) the same signal after 124 averages, showing clearly the smoother $100 \mathrm{~Hz}$ modulation structure of the lamp's intensity.

Light enters the device through a narrow solid angle collimator made of several $0.3 \mathrm{~mm}$ diameter apertures. Two interference filters $(10 \mathrm{~nm}$ bandpass, centered at $532 \mathrm{~nm})$ inserted before and after the apertures prevent overloading of the PM. The apertures and filters are held in a removable collimator block [7] that is mounted onto the PM using a Hamamatsu type A10030 adapter block ${ }^{6}$. The collimator, PM and batteries are contained in a handheld housing $(210 \times$ $100 \times 30 \mathrm{~mm}^{3}$ ). Two potentiometers allow individual control of the PM's high voltage and the discriminator level. Electric pulses produced by individual photons in the PM are rendered acoustically by a small loudspeaker built into the housing. Output connectors allow the pulse signals to be displayed by an external amplifier/loudspeaker system, an oscilloscope or to be further processed by pulse counting electronics.

Figures 1(b) and (c) show, respectively, a photograph of the device and of the printed circuit board of the control electronics. The device is designed to be kept on the left-hand side. A push button placed on the right-hand side of the box can be operated by the left-hand forefinger. In this way, the right-hand side is free to manipulate the high voltage and the discriminator level control knobs on the front panel. Photon pulses exceeding the discriminator level are sent to an LED on the front panel, whose blink rate thus reproduces the photon count rate. Two further LEDs on the lower right side indicate the 'on/off' status of the device and the charge level of the batteries. On the housing side opposite to the collimator entrance, three BNC connectors deliver the analogue PM pulses, the TTL pulses and the discriminator level, respectively. In this way, the relevant signals can be connected to further processing electronics

\footnotetext{
${ }^{6}$ http://jp.hamamatsu.com/products/sensor-etd/3006/A10030-01/index_en .html
}

(such as an oscilloscope connected to a multimedia projector or an external audio system) that allows demonstrations in front of a large public. By means of two screws, the collimator can be easily replaced by a collimator containing filters for the spectral range.

\section{Experimental results and discussion}

Figure 2 shows some typical oscilloscope traces.

Besides its use as a standalone device that demonstrates photon pulses, the device has a number of other interesting applications in teaching: When using a collimator block with interference filters that pass only a specific spectral line one can demonstrate the remote sensing of chemical elements, such as the presence of mercury (identified, e.g., by its $546.1 \mathrm{~nm}$ green line) in the light from a fluorescence tube or a street lamp (see figure 3).

When pointing the device at a distant surface illuminated by an enlarged laser spot, one may demonstrate the proportionality relation between the brightness of the spot and the photon click rate. When increasing the incident radiation such that the average photon rate exceeds the inverse of their pulse widths, the individual pulses pile up to form a fluctuating continuous signal that is well suited for illustrating the concept of shot noise and for making quantitative measurements thereof.

\section{Summary}

We have presented a simple handheld device that allows us to illustrate the discrete nature of light by rendering PM pulses produced by individual photons acoustically or as oscilloscope traces. 


\section{Acknowledgment}

The authors acknowledge financial support from the Scopes program (grant no. IZ73Z0-127942-1) of the Swiss National Science Foundation.

\section{References}

[1] Huygens C 2005 Treatise on Light (Chicago, IL: University of Chicago Press) (Engl. transl.) (http://www.gutenberg. org/files/14725/14725-h/14725-h.htm)

[2] Newton I 1730 Opticks: or, a Treatise of the Reflections, Refractions, Inflexions and Colours of Light 4 th edn
(London: W Innys) (http://www.gutenberg.org/files/33504/ 33504-h/33504-h.htm)

[3] Kumar M 2010 Quantum: Einstein, Bohr, and the Great Debate about the Nature of Reality (Cambridge: Icon Books)

[4] Lamb W E Jr 1995 Anti-photon Appl. Phys. B 60 77-84

[5] Dimitrova T L and Weis A 2008 The wave-particle duality of light: a demonstration experiment Am. J. Phys. 76 137-42

[6] Dimitrova T L and Weis 2010 Single photon quantum erasing: a demonstration experiment Eur. J. Phys. 31 625-37

[7] Dimitrova T L and Weis A 2011 A portable double slit quantum eraser with individual photons Eur. J. Phys. 32 1535-46 